\title{
Molecular phylogeny and morphological revision of the Ctenotus labillardieri (Reptilia: Squamata: Scincidae) species group and a new species of immediate conservation concern in the southwestern Australian biodiversity hotspot
}

\author{
GEOFFREY M. KAY \& J. SCOTT KEOGH ${ }^{1}$ \\ Division of Evolution, Ecology and Genetics, Research School of Biology, The Australian National University, Canberra, ACT 0200 , \\ Australia \\ ${ }^{\text {l} C o r r e s p o n d i n g ~ a u t h o r . ~ E-m a i l: ~ S c o t t . K e o g h @ a n u . e d u . a u ~}$
}

\begin{abstract}
Ctenotus is the largest and most diverse genus of skinks in Australia with at least 97 described species. We generated large mitochondrial and nuclear DNA data sets for 70 individuals representing all available species in the $C$. labillardieri speciesgroup to produce the first comprehensive phylogeny for this clade. The widespread $C$. labillardieri was sampled extensively to provide the first detailed phylogeographic data set for a reptile in the southwestern Australian biodiversity hotspot. We supplemented our molecular data with a comprehensive morphological dataset for the entire group, and together these data are used to revise the group and describe a new species. The morphologically highly variable species $C$. labillardieri comprises seven well-supported genetic clades that each occupy distinct geographic regions. The phylogeographic patterns observed in this taxon are consistent with studies of frogs, plants and invertebrates, adding strength to emerging biogeographic hypotheses in this iconic region. The species $C$. catenifer, $C$. youngsoni, and $C$. gemmula are well supported, and despite limited sampling both $C$. catenifer and $C$. gemmula show substantial genetic structure. The threatened $C$. lancelini from Lancelin Island and the adjacent mainland is the sister taxon to a new species from the Swan Coastal Plain, which we describe as $C$. ora $\mathbf{s p . ~ n o v . ~ T h i s ~}$ species is a habitat specialist, occurring primarily in sandy regions south of Perth that currently are under intense development. Ctenotus ora sp. nov. should be considered for conservation attention immediately.
\end{abstract}

Key words: cryptic species, lizard, skink, ND2, ATP, southwestern Australia, biodiversity hotspot, Swan Coastal Plain

\section{Introduction}

Southwestern Australia (SWA) is an iconic region, increasingly renowned for its high levels of biodiversity and endemism. It is recognized as one of the world's top 25 "biodiversity hotspots" (Cincotta et al. 2000; Myers et al. 2000), based largely on its highly diverse and endemic flora (Beard et al. 2000). More recent phylogeographic studies on SWA plants have demonstrated that plant genetic diversity is even more extreme than previously thought, with current taxonomy a gross underestimate of the true diversity ( Byrne et al. 2003a,b; Hopper \& Gioia 2004; Byrne \& Hines 2004; Byrne 2007; Byrne \& Hopper 2008;). A similar theme is emerging from genetic studies on SWA animals, including frogs (Driscoll 1998a,b; Berry 2001; Edwards 2007a,b; Edwards et al. 2007, 2008; Morgan et al. 2007), and invertebrates (Main 1996, 1999; Horwitz \& Adams 2000; Gouws et al. 2006; Cooper et al. 2011; Rix \& Harvey 2012).

Combining what is know about phylogeographic patterns of SWA taxa, it appears that major climatic and physical features occurring across SWA have been important in regional diversification. Climatic features, including strong rainfall and temperature gradients, have provided barriers to gene flow between Hopper's (1979) High Rainfall Zone (HRZ) and Transitional Rainfall Zone (TRZ) of SWA (Dodson \& Macphail 2004; Hopper \& Gioia 2004) (Figure 1). Furthermore, physical barriers including the subdued mountains of the Stirling Ranges and Darling Scarp, and granite outcrops, seem important for diversification (Wheeler \& Byrne 2006; Yates et al. 2007; Byrne \& Hopper 2008; Rix \& Harvey 2012), especially in coastal areas subject to eustatic sea-level fluctuations (Rabosky et al. 2004; Edwards 2008). Southwestern Australia also has been divided into a number of biogeographic subregions (Interim Biogeographic Regionalisation of Australia [IBRA]) based on broad landscape distinc- 
tions including attributes of climate, geomorphology, landform and biota (Environment Australia 2004). In SWA, these subregions are characterised by narrow, coastal zones, including the Swan Coastal Plain, Warren, and two divisions of the Esperance Plains, while adjacent inland areas comprise more extensive Jarrah and Mallee subregions. Understanding the patterns, and the potential environmental drivers, of diversification in SWA is critical in the identification and conservation of genetic diversity within this biodiversity hotspot.

Ctenotus Storr 1964 is the largest and most diverse genus of skinks in Australia with at least 97 described species (Wilson \& Swan 2008). A recently published molecular phylogeny for the genus identified a number of species groups within Ctenotus and highlighted their explosive diversification (Rabosky et al. 2007). Ctenotus labillardieri Duméril \& Bibron is a divergent sister lineage to all other Ctenotus in their phylogeny. Detailed morphological work on specimens morphologically very similar to Ctenotus labillardieri resulted in the description of several species that together were considered part of the 'labillardieri group' based on this similarity: C. catenifer Storr 1974, C. delli Storr 1974, C. gemmula Storr 1974, C. labillardieri, C. lancelini Ford 1969, and C. youngsoni Storr 1975 (Storr 1974; Storr, Smith, \& Johnstone 1999). While Storr did not intend to necessarily imply monophyly of the species he included in this group, a close relationship is likely given the morphological similarity of distribution (and is supported in our study). While Rabosky et al. (2007) found C. youngsoni to group with more distantly related Ctenotus, recent genetic work suggests it is closely related to C. labillardieri and allies (D. Rabosky, pers. comm.).

We generated mitochondrial and nuclear DNA data for all available species in the C. labillardieri species group to generate the first comprehensive phylogeny for this clade. The widespread $C$. labillardieri was sampled extensively to provide the first detailed phylogeographic data set for a reptile in SWA. We supplemented our molecular data with a comprehensive morphological data set for the entire group and these data together are used to revise the group and describe a new species of immediate conservation concern.

\section{Materials and methods}

Taxonomic sampling. Tissue samples were obtained for a total of 70 individuals (Table 1). These samples included all members of the $C$. labillardieri species group except for $C$. delli for which there was no available tissue. We sampled C. labillardieri thoroughly $(\mathrm{n}=57)$ to cover the full range of the species. Despite extensive fieldwork, we were able to obtain only a few samples between Albany and Esperance, which appears to be a genuine gap in the range of this species (Figure 1). This result is corroborated by the very low numbers of museum samples, supporting the hypotheses that populations are naturally sparse or highly localized in this region (Bush et al. 2007; Wilson \& Swan 2008).

\section{Molecular data and analyses}

Two molecular data sets were assembled to provide phylogenetically useful data at two hierarchical levels. Initially, a 1200 base-pair (bp) region of the mitochondrial genome was sequenced that included the entire mitochondrial NADH dehydrogenase subunit 2 (ND2) gene and part of the flanking tRNA-Methionine and tRNAAsparagine regions. This region was sequenced in all 70 animals used in this study, and formed the basis for preliminary assessment of phylogenetic relationships of species in the C. labillardieri species group, as well as the detailed phylogeographic analysis for $C$. labillardieri. This gene has been used in a wide range of vertebrate taxa, including a number of Australian reptiles and amphibians at comparable taxonomic levels (i.e., Edwards 2007a; Edwards et al. 2007, 2008; Morgan et al. 2007; Pepper et al. 2006, 2008). For the same 70 individuals we then generated a nDNA data set comprised of 550bp of the gene ATP (Skinner 2007), a gene that has proven particularly useful in Ctenotus (Rabosky et al. 2007).

Amplification of the mitochondrial DNA fragments was conducted following the methods of Pepper et al. (2006). Amplification of nuclear genomic DNA was conducted using an activation step at $94^{\circ} \mathrm{C}$ for 4 min, 14 cycles of denaturation at $94^{\circ} \mathrm{C}$ for $30 \mathrm{~s}$, annealing at $60^{\circ} \mathrm{C}$ for $25 \mathrm{~s}$ (stepping down to $55^{\circ} \mathrm{C}$ after 2 cycles and $50^{\circ} \mathrm{C}$ for the last 10 cycles), followed by 30 cycles of denaturation at $94^{\circ} \mathrm{C}$ for $30 \mathrm{~s}$, annealing at $46^{\circ} \mathrm{C}$ for $25 \mathrm{~s}$ and extension at $72^{\circ} \mathrm{C}$ for 90 s, with a final extension step at $72^{\circ} \mathrm{C}$ for $5 \mathrm{~min}$. Primer combinations used for PCR and sequencing are listed in Table 2. All PCR products were purified using an ammonium acetate clean-up method. Cycle-sequencing reactions were carried out following the methods of Pepper et al. (2006). Cleaned up sequence was run on an ABI 3100 auto-sequencer and edited sequences were aligned manually. 
TABLE 1. Locality information for all individuals sampled in this study. Museum ID\# refers to the voucher specimens held at the Western Australian Museum. Samples used in genetic (G) and morphological (M) analyses are noted.

\begin{tabular}{|c|c|c|c|c|c|}
\hline Taxon & Museum ID\# & $\begin{array}{l}\text { Latitude } \\
\text { (S) }\end{array}$ & $\begin{array}{l}\text { Longitude } \\
\text { (E) }\end{array}$ & Locality & Analyses \\
\hline \multirow[t]{16}{*}{ Ctenotus catenifer } & 68924 & -30.40000 & 115.45000 & Badgingarra National Park & M \\
\hline & 83015 & -35.00000 & 117.93330 & Emu Point, Albany & M \\
\hline & 83017 & -35.00000 & 117.93330 & Emu Point, Albany & M \\
\hline & 84505 & -34.99020 & 117.98060 & $2.5 \mathrm{~km} \mathrm{Ne}$ Mount Martin & M \\
\hline & 84508 & -34.99160 & 117.98330 & Mount Martin & $\mathrm{M}, \mathrm{G}$ \\
\hline & 90124 & -34.30000 & 115.17920 & $2.5 \mathrm{~km} \mathrm{Ne}$ Augusta & M \\
\hline & 90365 & -35.03160 & 116.85080 & Walpole-Nornalup National Park & M \\
\hline & 91155 & -34.40000 & 117.86670 & 4km E Talyuberlup Peak & M \\
\hline & 93732 & -33.71660 & 115.33333 & Ambergate Reserve & M \\
\hline & 117161 & -35.00000 & 117.95000 & Emu Point & $\mathrm{M}, \mathrm{G}$ \\
\hline & 121359 & -33.55000 & 115.05000 & Dunsborough & $\mathrm{M}, \mathrm{G}$ \\
\hline & 129085 & -34.41660 & 115.68330 & Lake Jasper & $\mathrm{M}, \mathrm{G}$ \\
\hline & 132008 & -33.82580 & 122.97360 & Cape Arid National Park & M \\
\hline & 135711 & -35.07300 & 117.62060 & Kronkup Tip & $\mathrm{M}, \mathrm{G}$ \\
\hline & 163142 & -35.05130 & 117.76060 & Albany Windfarm & M \\
\hline & 163143 & -35.05130 & 117.76060 & Albany Windfarm & M \\
\hline \multirow[t]{13}{*}{ Ctenotus delli } & 46191 & -31.96660 & 116.16670 & 1 1/2 Miles S Mundaring Weir & M \\
\hline & 49284 & -32.13330 & 116.30000 & Dale Forest Mt Dale Area & M \\
\hline & 51035 & -32.13330 & 116.30000 & Mt Dale & M \\
\hline & 56861 & -32.16660 & 116.25000 & Mt Dale, Brookton Hwy & M \\
\hline & 57719 & -31.90000 & 116.26670 & $7 \mathrm{~km}$ E Of Sawyers Valley & M \\
\hline & 74977 & -32.91660 & 116.21670 & Se Dwellingup. & M \\
\hline & 75878 & -32.91660 & 116.21670 & Se Dwellingup & M \\
\hline & 96280 & -32.05000 & 115.95000 & South Canning Catchment & M \\
\hline & 103739 & -32.51660 & 116.03330 & North Dandalup & M \\
\hline & 104373 & -32.53330 & 116.40000 & $7 \mathrm{~km}$ Nw Of North Bannister & M \\
\hline & 113444 & -32.51660 & 116.00000 & North Dandalup Dam & M \\
\hline & 144173 & -33.35630 & 116.22420 & 4km South Collie & M \\
\hline & 156643 & -33.13910 & 116.42890 & Collie Area & M \\
\hline \multirow[t]{18}{*}{ Ctenotus gemmula } & 59707 & -31.70000 & 115.93330 & Melaleuca Park & $\mathrm{M}$ \\
\hline & 77749 & -32.72500 & 125.03330 & Toolinna Rockhole & $\mathrm{M}, \mathrm{G}$ \\
\hline & 91049 & -34.43330 & 117.88330 & 7km Se Talyuberlup Peak & M \\
\hline & 94104 & -32.72500 & 125.03330 & 6km Ne Toolinna Rockhole & M \\
\hline & 94475 & -33.83330 & 119.83330 & Fitzgerald River National Park & M \\
\hline & 94675 & -34.13330 & 119.45000 & Fitzgerald River National Park & M \\
\hline & 96257 & -33.45000 & 121.73330 & Scaddan & M \\
\hline & 100822 & -33.81660 & 121.13330 & Stokes National Park & M \\
\hline & 103345 & -30.61660 & 115.41670 & Cooljarloo & M \\
\hline & 116529 & -33.65000 & 120.43330 & $5.5 \mathrm{~km}$ E Bandalup Hill & $\mathrm{M}, \mathrm{G}$ \\
\hline & 119462 & -33.73970 & 118.97440 & 18km Ssw Ravensthorpe & $\mathrm{M}, \mathrm{G}$ \\
\hline & 121402 & -33.95000 & 120.45000 & Mason Bay & M \\
\hline & 137202 & -34.88330 & 118.60000 & Stirling Range National Park & M \\
\hline & 140514 & -30.57440 & 115.34170 & Cataby & $\mathrm{M}, \mathrm{G}$ \\
\hline & 143067 & -33.61580 & 119.19940 & Lake Magenta Nr & M \\
\hline & 144223 & -33.57580 & 120.30750 & Bandalup Hill & $\mathrm{M}, \mathrm{G}$ \\
\hline & 150245 & -33.34750 & 120.87170 & Pyramid Lake Area & M \\
\hline & 156937 & -34.54550 & 118.53080 & Upper Kalgan & M \\
\hline
\end{tabular}


TABLE 1. (continued)

\begin{tabular}{|c|c|c|c|c|c|}
\hline Taxon & Museum ID\# & $\begin{array}{l}\text { Latitude } \\
\text { (S) }\end{array}$ & $\begin{array}{l}\text { Longitude } \\
\text { (E) }\end{array}$ & Locality & Analyses \\
\hline \multicolumn{6}{|c|}{ Ctenotus labillardieri } \\
\hline \multirow[t]{16}{*}{ Clade 1} & 90231 & -35.0333 & 117.4667 & Koirchekup Hill & $\mathrm{M}, \mathrm{G}$ \\
\hline & 97816 & -35.05 & 118.0333 & Michaelmas Island & $\mathrm{M}, \mathrm{G}$ \\
\hline & 97817 & -35.05 & 118.0333 & Michaelmas Island & $\mathrm{M}, \mathrm{G}$ \\
\hline & 115320 & -34.3 & 116.15 & Manjimup & $\mathrm{M}, \mathrm{G}$ \\
\hline & 116249 & -34.575 & 116.4 & Mt Burnside & $\mathrm{M}, \mathrm{G}$ \\
\hline & 116251 & -34.575 & 116.4 & Mt Burnside & $\mathrm{M}, \mathrm{G}$ \\
\hline & 117354 & -34.9333 & 116.45 & Broke Inlet & $\mathrm{M}, \mathrm{G}$ \\
\hline & 117357 & -34.95 & 117.0333 & Kent River Bridge & $\mathrm{M}, \mathrm{G}$ \\
\hline & 117359 & -34.9333 & 116.45 & Broke Inlet & $\mathrm{M}, \mathrm{G}$ \\
\hline & 129677 & -34.85 & 117.35 & Denmark & $\mathrm{M}, \mathrm{G}$ \\
\hline & 136391 & -35.0697 & 117.7814 & Albany & $\mathrm{M}, \mathrm{G}$ \\
\hline & 140763 & -34.9636 & 116.6028 & Walpole & $\mathrm{M}, \mathrm{G}$ \\
\hline & 142046 & -34.89039 & 118.40931 & Cheyne Beach & $\mathrm{M}, \mathrm{G}$ \\
\hline & 142938 & -34.89039 & 118.40931 & Cheyne Beach & $\mathrm{M}, \mathrm{G}$ \\
\hline & 165587 & -34.99 & 117.2819 & Walpole & $\mathrm{M}, \mathrm{G}$ \\
\hline & 165589 & -34.99 & 117.2819 & Walpole & $\mathrm{M}, \mathrm{G}$ \\
\hline \multirow[t]{2}{*}{ Clade 2} & 134072 & -33.74554 & 119.66578 & Fitzgerald Road & $\mathrm{G}$ \\
\hline & 142908 & -34.2166 & 119.4333 & West Mt Barren & $\mathrm{M}, \mathrm{G}$ \\
\hline \multirow[t]{14}{*}{ Clade 3} & 113336 & -33.9833 & 115.1167 & Margaret River & $\mathrm{M}, \mathrm{G}$ \\
\hline & 113337 & -33.9833 & 115.1167 & Margaret River & $\mathrm{M}, \mathrm{G}$ \\
\hline & 116229 & -33.875 & 116.2833 & Boyup Brook & $\mathrm{M}, \mathrm{G}$ \\
\hline & 116236 & -34.0166 & 115.7333 & Nannup & $\mathrm{M}, \mathrm{G}$ \\
\hline & 119037 & -33.35 & 116 & Wellington Dam & $\mathrm{M}, \mathrm{G}$ \\
\hline & 127458 & -34.3333 & 115.15 & Augusta & $\mathrm{M}, \mathrm{G}$ \\
\hline & 129644 & -34.4013 & 115.6436 & Lake Jasper & $\mathrm{M}, \mathrm{G}$ \\
\hline & 132092 & -34.3666 & 115.1333 & Cape Leeuwin & $\mathrm{M}, \mathrm{G}$ \\
\hline & 132093 & -34.3666 & 115.1333 & Cape Leeuwin & $\mathrm{M}, \mathrm{G}$ \\
\hline & 135756 & -34.1 & 115.0333 & Cape Leeuwin & $\mathrm{M}, \mathrm{G}$ \\
\hline & 140784 & -34.3833 & 115.15 & Augusta & $\mathrm{M}, \mathrm{G}$ \\
\hline & 142327 & -32.74642 & 116.15208 & Dwellingup & $\mathrm{M}, \mathrm{G}$ \\
\hline & 142382 & -32.74642 & 116.15208 & Dwellingup & $\mathrm{M}, \mathrm{G}$ \\
\hline & 165590 & -34.2386 & 115.6333 & Karridale & $\mathrm{M}, \mathrm{G}$ \\
\hline \multirow[t]{3}{*}{ Clade 4} & 129661 & -33.8333 & 121.2833 & Quagi Beach & $\mathrm{M}, \mathrm{G}$ \\
\hline & 129662 & -33.8333 & 121.2833 & Quagi Beach & $\mathrm{M}, \mathrm{G}$ \\
\hline & 134073 & -33.92337 & 120.03042 & East Mt Barren & $\mathrm{G}$ \\
\hline \multirow[t]{3}{*}{ Clade 5} & 142949 & -34.36938 & 118.24863 & Bluff Knoll & $\mathrm{M}, \mathrm{G}$ \\
\hline & DLE58 & -34.3699 & 118.24982 & Bluff Knoll & $\mathrm{G}$ \\
\hline & DLE59 & -34.3699 & 118.24982 & Bluff Knoll & $\mathrm{G}$ \\
\hline \multirow[t]{9}{*}{ Clade 6} & 97507 & -33.9 & 122.75 & Ben Island & $\mathrm{M}, \mathrm{G}$ \\
\hline & 97525 & -34.0333 & 123.2167 & Harlequin Island & M \\
\hline & 116228 & -33.875 & 116.2833 & Boyup Brook & M \\
\hline & 131903 & -33.9972 & 122.1194 & Mount Le Grand & $\mathrm{M}, \mathrm{G}$ \\
\hline & 131904 & -33.9972 & 122.1194 & Mount Le Grand & $\mathrm{M}, \mathrm{G}$ \\
\hline & 131908 & -34.0041 & 122.1722 & Hellfire Bay & $\mathrm{M}, \mathrm{G}$ \\
\hline & 131914 & -34.0041 & 122.1722 & Hellfire Bay & $\mathrm{M}, \mathrm{G}$ \\
\hline & 131916 & -33.9472 & 122.5667 & Mount Belches & $\mathrm{M}, \mathrm{G}$ \\
\hline & 131919 & -33.9472 & 122.5667 & Mount Belches & $\mathrm{M}, \mathrm{G}$ \\
\hline
\end{tabular}


TABLE 1. (continued)

\begin{tabular}{|c|c|c|c|c|c|}
\hline Taxon & Museum ID\# & $\begin{array}{l}\text { Latitude } \\
\text { (S) }\end{array}$ & $\begin{array}{l}\text { Longitude } \\
\text { (E) }\end{array}$ & Locality & Analyses \\
\hline & 131924 & -33.8166 & 122.2139 & Mount Merivale & $\mathrm{M}, \mathrm{G}$ \\
\hline & 131925 & -33.8166 & 122.2139 & Mount Merivale & $\mathrm{M}, \mathrm{G}$ \\
\hline & 131926 & -33.9722 & 122.1292 & Mount Le Grand & $\mathrm{M}, \mathrm{G}$ \\
\hline & 132164 & -33.8166 & 123.0333 & Thomas River & $\mathrm{M}, \mathrm{G}$ \\
\hline & 135665 & -34.1333 & 122.25 & Mondrain Island & $\mathrm{M}, \mathrm{G}$ \\
\hline & 135666 & -34.1333 & 122.25 & Mondrain Island & $\mathrm{M}, \mathrm{G}$ \\
\hline & 135758 & -34.1 & 115.0333 & Cape Leeuwin & M \\
\hline & 140529 & -34.0361 & 121.9917 & Sandy Hook Island & $\mathrm{M}, \mathrm{G}^{*}$ \\
\hline & 140530 & -34.0361 & 121.9917 & Sandy Hook Island & $\mathrm{M}, \mathrm{G}$ \\
\hline & 166084 & -31.88139 & 116.17806 & Stoneville & $\mathrm{M}, \mathrm{G}$ \\
\hline Clade 7 & 166085 & -31.88139 & 116.17806 & Stoneville & M, G \\
\hline \multirow[t]{11}{*}{ Ctenotus lancelini } & 18871 & -31.00000 & 115.31670 & Lancelin Island & $\mathrm{M}$ \\
\hline & 18873 & -31.00000 & 115.31670 & Lancelin Island & M \\
\hline & 18874 & -31.00000 & 115.31670 & Lancelin Island & M \\
\hline & 18875 & -31.00000 & 115.31670 & Lancelin Island & M \\
\hline & 50126 & -31.00000 & 115.31670 & Lancelin Island & M \\
\hline & 52098 & -31.00000 & 115.31670 & Lancelin Island & M \\
\hline & 52099 & -31.00000 & 115.31670 & Lancelin Island & M \\
\hline & 52100 & -31.00000 & 115.31670 & Lancelin Island & M \\
\hline & 121883 & -31.01660 & 115.33330 & Lancelin & $\mathrm{M}, \mathrm{G}$ \\
\hline & 125723 & -31.00000 & 115.31670 & Lancelin Island & $\mathrm{M}, \mathrm{G}$ \\
\hline & 125724 & -31.00000 & 115.31670 & Lancelin Island & $\mathrm{M}, \mathrm{G}$ \\
\hline \multirow[t]{17}{*}{ Ctenotus youngsoni } & 54800 & -26.38333 & 113.31667 & False Entrance Well & $\mathrm{M}$ \\
\hline & 54825 & -26.38333 & 113.31667 & False Entrance Well & M \\
\hline & 57089 & -26.13333 & 113.21667 & Dirk Hartog Island & M \\
\hline & 64426 & -26.90000 & 113.71667 & $19 \mathrm{~km}$ South Tamala & M \\
\hline & 66208 & -26.38333 & 113.31667 & False Entrance Well & M \\
\hline & 66209 & -26.38333 & 113.31667 & False Entrance Well & M \\
\hline & 66293 & -26.15000 & 113.16667 & Steep Point & M \\
\hline & 81331 & -26.43333 & 113.30000 & Zuytdorp Point & M \\
\hline & 81332 & -26.43333 & 113.30000 & Zuytdorp Point & M \\
\hline & 82779 & -26.40000 & 113.30000 & Zuytdorp Point & M \\
\hline & 91692 & -26.40000 & 113.30000 & False Entrance Well & M \\
\hline & 103227 & -26.55000 & 113.76667 & Salutation Island & M \\
\hline & 103234 & -26.55000 & 113.65000 & Three Bays Island & M \\
\hline & 113648 & -26.00000 & 113.20000 & Dirk Hartog Island & G \\
\hline & 113664 & -25.93333 & 113.16667 & Dirk Hartog Island & G \\
\hline & 135504 & -26.38333 & 113.31667 & False Entrance Well & $\mathrm{M}, \mathrm{G}$ \\
\hline & 135533 & -26.38333 & 113.31667 & False Entrance Well & G \\
\hline \multirow[t]{5}{*}{ Ctenotus ora sp. nov. } & 73591 & -32.83330 & 115.65000 & Yalgorup National Park & $\mathrm{M}$ \\
\hline & 81601 & -33.35000 & 115.70000 & Eaton & M \\
\hline & 119059 & -32.66660 & 115.71670 & Pinjarra & $\mathrm{M}, \mathrm{G}$ \\
\hline & 131983 & -33.53910 & 115.02030 & Cape Naturaliste & $\mathrm{M}, \mathrm{G}$ \\
\hline & 141244 & -33.63330 & 115.03750 & Yallingup Brook & M, G \\
\hline
\end{tabular}

Individual data sets were created for each gene, and we used unweighted heuristic parsimony and Bayesian approaches to analyze the data. Unweighted heuristic parsimony analyses were implemented with the computer program PAUP* (Swofford 2002). We used TBR branch swapping and ran the parsimony analysis five times from random starting points to make sure overall tree space was well searched. Bayesian analyses were implemented 
with the computer program MrBayes (v3.0b4: Huelsenbeck \& Ronquist 2001). We performed individual Bayesian analyses on each gene with the nDNA treated as a single unit and the mtDNA gene divided into four partitions: $1^{\text {st }}$, $2^{\text {nd }}, 3^{\text {rd }}$ codon positions for the coding region, and the tRNA. We allowed all parameters to be estimated from the data during the run. We used the default value of four Markov chains per run and also ran the full analysis two times to make sure overall tree-space was well sampled and to avoid getting trapped in local optima. We ran each analysis for a total of 4,000,000 generations and sampled the chain after every 100 generations, resulting in 40,000 sampled trees. Log-likelihood values reached a plateau after approximately 100,000 generations $(1,000$ sampled trees), so to make sure that we discarded the full burn-in phase, we discarded the first 10,000 trees and used the last 30,000 trees to estimate Bayesian posterior probabilities. Ctenotus robustus was used as the outgroup in phylogenetic analyses based on the results of Rabosky et al. (2007). We also created a concatenated data set using both genes and implemented a partitioned Bayesian analysis as above, but with five partitions: $1^{\text {st }}, 2^{\text {nd }}, 3^{\text {rd }}$ codon positions for the coding region of ND2; tRNA, and nDNA. For each analysis we used the results of 10,000 unweighted parsimony bootstrap replicates and Bayesian posterior probabilities to assess branch support.

TABLE 2. Amplification and sequencing primers (5' to 3') used in this study. Modified (Mod.) primers are noted.

\begin{tabular}{llll}
\hline Gene & Name & Sequence & Source \\
\hline tRNA-'sn & tRNA-'ss & 5'-CTAAAATRTTRCGGGATCGAGGCC-3' & Read et al., 2001 \\
tRNA-' ${ }^{\text {Met }}$ & L4437 & 5'-AAGCTTTCGGGGCCCATACC-3' & Macey et al., 1998 \\
ND2 & L4882 & 5'-CAACATGACAAAAAATCGCCCC-3' & Macey et al., 2000 \\
ND2 & AT4882 & 5'-CAACATGACAAAAATTRGCCCC-3' & Macey et al., 2000 (mod.) \\
ND2 & CM4882 & 5'-CYACATGACAAAAAATTGCACC-3' & Macey et al., 2000 (mod.) \\
ATP & G613R & 5'-TCTGTCCATAAACTAGCG-3' & Skinner, 2007 (mod.) \\
ATP & ATPSB1 & 5'-CGTGAGGGHAAYGATTTHTACCATGA-3' & Skinner, 2007 (mod.) \\
\hline
\end{tabular}

\section{Morphological data analyses}

We carried out a detailed assessment of morphological variation in the species group by examination of all available specimens housed in the Western Australian Museum that were included in the molecular phylogeny, as well as additional museum specimens where genetic sample sizes were low or where additional information was required (Table 1). Our morphological data set was based on the characters identified in the comprehensive morphological revision of the group by Storr (1974). Table 3 presents the morphological characters measured and their descriptions. In total 19 characters were measured in 133 specimens. Measurements were made with electronic callipers to the nearest $1 \mathrm{~mm}, 0.5 \mathrm{~mm}$ or $0.1 \mathrm{~mm}$ as appropriate. We analyzed the 10 continuous charaters (see Table 3 ) with Principal Components Analysis (PCA), which does not identify groups a priori, to summarize inter-specific variation in these characters. We calculated standard principal components with variance-covariance and imputation of any missing data so we could include all individuals (although very little was missing).

\section{Results}

Molecular data. Full details of collection localities and voucher information for each individual are given in Table 1. Alignment of both the mtDNA and nDNA fragments was straightforward. The ND2 alignment comprised 1133 characters of which $353(31 \%)$ were parsimony informative. The nuclear ATP gene alignment comprised 477 characters of which 33 (6.9\%) were parsimony informative. Uncorrected "p" genetic distances in the mtDNA data set are summarized in Tables 4 and 5. 
TABLE 3. Morphological characters and their abbreviations used in this study.

\begin{tabular}{|c|c|}
\hline Character & Description \\
\hline \multicolumn{2}{|c|}{ Continuous characters } \\
\hline SVL & Snout-vent length \\
\hline HeadL & Head length from tip of snout posterior of quadrate bone \\
\hline HLL & Hind-limb length of fully extended limb, including claw \\
\hline FLL & Fore-limb length of fully extended limb, including claw \\
\hline ILL & Interlimb distance measured from posterior margin of forelimb to anterior margin of hindlimb \\
\hline TailL & Tail length from vent to tip (original tails only) \\
\hline LorW:H & The height to width ratio of the second loreal scale \\
\hline MidB & Number of scales in the mid-body scale row in the middle of the body \\
\hline Lam4Toe & Number of rows of lamellae under the $4^{\text {th }}$ toe on one foot \\
\hline VentS & Number of ventral scales counted from forelimb to vent \\
\hline \multicolumn{2}{|c|}{ Categorical characters } \\
\hline NasSS & Nasal scale separation scored as separated $(\mathrm{S})$ or in contact $(\mathrm{C})$ \\
\hline PrefSS & Prefrontal scale separation scored as separated $(\mathrm{S})$ or in contact $(\mathrm{C})$ \\
\hline SOS & Number of supraocular scales \\
\hline SOSContact & Number of supraocular scales in contact with frontal scale $(2,3$ or 4$)$ \\
\hline SupCil & $\begin{array}{l}\text { Number of supracilaries, beginning with the scale adjoining the prefrontal and loreal, and ending with the } \\
\text { scale still contacting cilaries and last supraocular }(7,8 \text { or } 9)\end{array}$ \\
\hline Palp & Number of palpebral scales $(8,9,10,11,12$ or 13$)$ \\
\hline UppLab & Number of upper labial scales $(7,8$ or 9$)$ \\
\hline EarL & Number of ear lobule scales $(3,4$ or 5$)$ \\
\hline EarLPos & The relative position of the largest ear lobule ( 1 - highest in ear, 2, 3, or 4 - lowest) \\
\hline
\end{tabular}

TABLE 4. Ranges of intra-clade uncorrected "p" genetic distances for the C. labillardieri species group.

\begin{tabular}{ll}
\hline Taxon & Uncorrected "p" Genetic Distance \\
\hline Ctenotus catenifer & $0.0080-0.0507$ \\
C. gemmula & $0.0009-0.0630$ \\
C. labillardieri & $0-0.0773$ \\
C. labillardieri Clade 1 & $0-0.0221$ \\
C. labillardieri Clade 2 & 0.0063 \\
C. labillardieri Clade 3 & $0.0009-0.0257$ \\
C. labillardieri Clade 4 & $0.0044-0.0305$ \\
C. labillardieri Clade 5 & $0.0000-0.0090$ \\
C. labillardieri Clade 6 & $0.0000-0.0453$ \\
C. labillardieri Clade 7 & 0.0232 \\
C. lancelini & 0 \\
C. youngsoni & $0.0009-0.0063$ \\
C. ora sp. nov. & $0-0.0098$ \\
\hline
\end{tabular}

Table 5. Ranges of inter-clade uncorrected "p" genetic distances for the C. labillardieri species group.

\begin{tabular}{|c|c|c|c|c|c|c|c|c|c|c|c|}
\hline Taxon & C. catenifer & C. ora sp. nov. & C. gemmula & $\begin{array}{c}C . \\
\text { labillardieri } \\
\text { Clade } 1\end{array}$ & $\begin{array}{c}C . \\
\text { labillardieri } \\
\text { Clade } 2\end{array}$ & $\begin{array}{c}C . \\
\text { labillardieri } \\
\text { Clade } 3\end{array}$ & $\begin{array}{c}C . \\
\text { labillardieri } \\
\text { Clade } 4\end{array}$ & $\begin{array}{c}C . \\
\text { labillardieri } \\
\text { Clade } 5\end{array}$ & $\begin{array}{c}C . \\
\text { labillardieri } \\
\text { Clade } 6\end{array}$ & $\begin{array}{c}C . \\
\text { labillardieri } \\
\text { Clade } 7\end{array}$ & C. Iancelini \\
\hline C. ora sp. nov. & $0.1538-0.1679$ & & - & Crave 1 & Craue 2 & Ciac & Cract & Cide & hac & Co & - \\
\hline C. gemmula & $0.1412-0.1574$ & $0.1252-0.1315$ & . & . & . & . & . & . & . & . & . \\
\hline C. labillardieri Clade 1 & $0.1474-0.1600$ & $0.1093-0.1199$ & $0.1162-0.1364$ & - & - & . & - & - & . & . & - \\
\hline C. labillardieri Clade 2 & $0.1528-0.1574$ & $0.1110-0.1169$ & $0.1215-0.1275$ & $0.0177-0.0296$ & . & . & . & . & . & . & . \\
\hline C. labillardieri Clade 3 & $0.1439-0.1573$ & $0.1030-0.1164$ & $0.1136-0.1242$ & $0.0337-0.0471$ & $0.0275-0.0350$ & . & . & . & . & . & . \\
\hline C. labillardieri Clade 4 & $0.1463-0.1635$ & $0.1094-0.1208$ & $0.1194-0.1358$ & $0.0386-0.0580$ & $0.0368-0.0548$ & $0.0458-0.0621$ & . & . & . & . & . \\
\hline C. labillardieri Clade 5 & $0.1483-0.1536$ & $0.1037-0.1103$ & $0.1233-0.1278$ & $0.0440-0.0541$ & $0.0402-0.0431$ & $0.0465-0.0532$ & $0.0484-0.0574$ & . & . & . & . \\
\hline C. labillardieri Clade 6 & $0.1475-0.1601$ & $0.1164-0.1244$ & $0.1296-0.1376$ & $0.0568-0.0719$ & $0.0549-0.0648$ & $0.0613-0.0773$ & $0.0601-0.0746$ & $0.0610-0.0702$ & . & . & . \\
\hline C. labillardieri Clade 7 & $0.1456-0.1552$ & $0.1142-0.1238$ & $0.1248-0.1433$ & $0.0615-0.0727$ & $0.0606-0.0682$ & $0.0623-0.0694$ & $0.0636-0.0739$ & $0.0616-0.0672$ & $0.0563-0.0687$ & . & : \\
\hline C. lancelini & $0.1610-0.1689$ & $0.0426-0.0453$ & $0.1288-0.1350$ & $0.1057-0.1146$ & $0.1078-0.1084$ & $0.1021-0.1084$ & $0.1112-0.1190$ & $0.1049-0.1057$ & $0.1111-0.1209$ & $0.1169-0.1229$ & . \\
\hline C. youngsoni & $0.1677-0.1738$ & $0.1721-0.1780$ & $0.1622-0.1753$ & $0.1572-0.1737$ & $0.1601-0.1697$ & $0.1601-0.1697$ & $0.1537-0.1693$ & $0.1572-0.1631$ & $0.1686-0.1788$ & $0.1561-0.1674$ & $0.1693-0.1707$ \\
\hline
\end{tabular}


Table 6. Summary statistics for the morphological data collected on members of the Ctenotus labillardieri group (mean \pm SD and range). See Table 2 for character definitions and Materials and Methods for details of statistical tests.

\begin{tabular}{|c|c|c|c|c|c|c|c|}
\hline Character & C. catenifer & C. delli & C. gemmula & C. labillardieri & C. lancelini & C. ora sp. nov. & C. younsoni \\
\hline $\mathrm{n}$ & 16 & 14 & 18 & 54 & 11 & 5 & 14 \\
\hline SVL & $\begin{array}{c}49.44 \pm 2.44 \\
(41-56)\end{array}$ & $\begin{array}{c}49.36 \pm 2.60 \\
(38-59)\end{array}$ & $\begin{array}{c}48.17 \pm 2.30 \\
(29-59)\end{array}$ & $\begin{array}{c}58.70 \pm 1.33 \\
(29-75)\end{array}$ & $\begin{array}{c}61.91 \pm 2.94 \\
(30-78)\end{array}$ & $\begin{array}{c}47.40 \pm 4.36 \\
(33-60)\end{array}$ & $\begin{array}{c}72.71 \pm 2.61 \\
(56-83)\end{array}$ \\
\hline HeadL & $\begin{array}{c}11.51 \pm 0.38 \\
(8.9-12.7)\end{array}$ & $\begin{array}{c}11.81 \pm 0.41 \\
(8.9-13.4)\end{array}$ & $\begin{array}{c}11.13 \pm 0.36 \\
(8.1-12.5)\end{array}$ & $\begin{array}{c}12.79 \pm 0.21 \\
(8.3-18.3)\end{array}$ & $\begin{array}{c}13.27 \pm 0.46 \\
(8.6-15.3)\end{array}$ & $\begin{array}{c}11.88 \pm 0.69 \\
(9.0-14.4)\end{array}$ & $\begin{array}{c}15.76 \pm 0.41 \\
(13.7-17.0)\end{array}$ \\
\hline HLL & $\begin{array}{c}18.94 \pm 0.83 \\
(16-21)\end{array}$ & $\begin{array}{c}20.21 \pm 0.89 \\
(16-24)\end{array}$ & $\begin{array}{c}20.22 \pm 0.78 \\
(15-29)\end{array}$ & $\begin{array}{c}23.61 \pm 0.45 \\
(13-30)\end{array}$ & $\begin{array}{c}24.64 \pm 1.00 \\
(14-30)\end{array}$ & $\begin{array}{c}19.60 \pm 1.49 \\
(15-23)\end{array}$ & $\begin{array}{c}30.07 \pm 0.89 \\
(25-34)\end{array}$ \\
\hline FLL & $\begin{array}{c}12.38 \pm 0.53 \\
(9-14)\end{array}$ & $\begin{array}{c}12.64 \pm 0.57 \\
(9-16)\end{array}$ & $\begin{array}{c}12.61 \pm 0.50 \\
(10-15)\end{array}$ & $\begin{array}{c}14.94 \pm 0.29 \\
(8-18)\end{array}$ & $\begin{array}{c}15.09 \pm 0.64 \\
(10-18)\end{array}$ & $\begin{array}{c}13.60 \pm 0.95 \\
(11-18)\end{array}$ & $\begin{array}{c}18.93 \pm 0.57 \\
(15-28)\end{array}$ \\
\hline ILL & $\begin{array}{c}27.89 \pm 1.61 \\
(22-35)\end{array}$ & $\begin{array}{c}27.36 \pm 1.72 \\
(18-39)\end{array}$ & $\begin{array}{c}27.25 \pm 1.52 \\
(15-35)\end{array}$ & $\begin{array}{c}31.56 \pm 0.88 \\
(14-44)\end{array}$ & $\begin{array}{c}40.10 \pm 1.94 \\
(17-52)\end{array}$ & $\begin{array}{c}27.04 \pm 2.88 \\
(18-35)\end{array}$ & $\begin{array}{c}40.79 \pm 1.72 \\
(30-53)\end{array}$ \\
\hline TailL & $\begin{array}{c}83.25 \pm 5.89 \\
(66-101)\end{array}$ & $\begin{array}{c}92.00 \pm 6.15 \\
(63-141)\end{array}$ & $\begin{array}{c}85.80 \pm 5.27 \\
(51-113)\end{array}$ & $\begin{array}{c}98.14 \pm 4.35 \\
(45-130)\end{array}$ & $\begin{array}{c}107.86 \pm 7.71 \\
(60-142)\end{array}$ & $\begin{array}{c}78.67 \pm 11.78 \\
(65-88)\end{array}$ & $\begin{array}{c}124.63 \pm 7.21 \\
(108-149)\end{array}$ \\
\hline LorH:W & $\begin{array}{l}0.72 \pm 0.03 \\
(0.41-1.00)\end{array}$ & $\begin{array}{l}0.77 \pm 0.03 \\
(0.63-0.94)\end{array}$ & $\begin{array}{l}0.73 \pm 0.03 \\
(0.44-0.91)\end{array}$ & $\begin{array}{l}0.69 \pm 0.02 \\
(0.46-0.92)\end{array}$ & $\begin{array}{l}0.76 \pm 0.04 \\
(0.63-1.00)\end{array}$ & $\begin{array}{l}0.81 \pm 0.05 \\
(0.73-0.91)\end{array}$ & $\begin{array}{l}0.68 \pm 0.03 \\
(0.59-0.83)\end{array}$ \\
\hline MidB & $\begin{array}{c}25.13 \pm 0.36 \\
\quad(22-28)\end{array}$ & $\begin{array}{c}26.86 \pm 0.39 \\
(24-30)\end{array}$ & $\begin{array}{c}24.00 \pm 0.34 \\
(22-26)\end{array}$ & $\begin{array}{c}26.04 \pm 0.20 \\
(23-29)\end{array}$ & $\begin{array}{c}22.73 \pm 0.44 \\
\quad(20-24)\end{array}$ & $\begin{array}{c}22.80 \pm 0.65 \\
\quad(21-24)\end{array}$ & $\begin{array}{c}28.50 \pm 0.39 \\
(26-32)\end{array}$ \\
\hline Lam4Toe & $\begin{array}{c}22.67 \pm 0.48 \\
(19-24)\end{array}$ & $\begin{array}{c}23.46 \pm 0.51 \\
(20-26)\end{array}$ & $\begin{array}{c}23.11 \pm 0.44 \\
(20-26)\end{array}$ & $\begin{array}{c}23.83 \pm 0.26 \\
(19-28)\end{array}$ & $\begin{array}{c}23.11 \pm 0.62 \\
(22-25)\end{array}$ & $\begin{array}{c}24.20 \pm 0.83 \\
(23-27)\end{array}$ & $\begin{array}{c}21.14 \pm 0.49 \\
\quad(18-23)\end{array}$ \\
\hline VentS & $\begin{array}{c}42.63 \pm 0.74 \\
(37-50)\end{array}$ & $\begin{array}{c}43.43 \pm 0.79 \\
(39-49)\end{array}$ & $\begin{array}{c}41.83 \pm 0.70 \\
(37-46)\end{array}$ & $\begin{array}{c}45.34 \pm 0.41 \\
(40-54)\end{array}$ & $\begin{array}{c}43.18 \pm 0.89 \\
(41-47)\end{array}$ & $\begin{array}{c}43.40 \pm 1.37 \\
(41-48)\end{array}$ & $\begin{array}{c}42.21 \pm 0.79 \\
(41-46)\end{array}$ \\
\hline NasSS & $Y(100 \%)$ & $\begin{array}{l}N(14 \%) \\
Y(86 \%)\end{array}$ & $Y(100 \%)$ & $Y(100 \%)$ & $Y(100 \%)$ & $Y(100 \%)$ & N (100\%) \\
\hline PrefSS & $Y(100 \%)$ & $\begin{array}{l}N(7 \%) \\
Y(93 \%)\end{array}$ & $\begin{array}{l}N(11 \%) \\
Y(89 \%)\end{array}$ & $\begin{array}{l}\text { N }(11 \%) \\
Y(89 \%)\end{array}$ & $Y(100 \%)$ & $Y(100 \%)$ & $\begin{array}{l}N(36 \%) \\
Y(64 \%)\end{array}$ \\
\hline sos & $\begin{array}{c}4(100 \%) \\
2(87 \%)\end{array}$ & $\begin{array}{c}4(100 \%) \\
2(79 \%)\end{array}$ & $4(100 \%)$ & $\begin{array}{c}4(100 \%) \\
2(92 \%)\end{array}$ & $4(100 \%)$ & $4(100 \%)$ & $4(100 \%)$ \\
\hline SosContact & $\begin{array}{l}3(6 \%) \\
4(6 \%)\end{array}$ & $\begin{array}{l}3(14 \%) \\
4(7 \%)\end{array}$ & $2(100 \%)$ & $\begin{array}{l}3(4 \%) \\
4(4 \%)\end{array}$ & $\begin{array}{c}2(91 \%) \\
3(9 \%)\end{array}$ & $2(100 \%)$ & $2(100 \%)$ \\
\hline SupCil & $\begin{array}{l}7(88 \%) \\
8(12 \%)\end{array}$ & $\begin{array}{l}7(58 \%) \\
8(42 \%)\end{array}$ & $\begin{array}{l}7(76 \%) \\
8(24 \%)\end{array}$ & $\begin{array}{c}7(49 \%) \\
8(49 \%) \\
9(2 \%)\end{array}$ & $\begin{array}{l}7(67 \%) \\
8(33 \%)\end{array}$ & $\begin{array}{l}7(40 \%) \\
8(60 \%)\end{array}$ & $\begin{array}{l}7(62 \%) \\
8(38 \%)\end{array}$ \\
\hline Palp & $\begin{array}{c}8(10 \%) \\
9(20 \%) \\
11(70 \%)\end{array}$ & $\begin{array}{c}9(14 \%) \\
10(14 \%) \\
11(43 \%) \\
12(29 \%)\end{array}$ & $\begin{array}{c}8(8 \%) \\
9(29 \%) \\
10(14 \%) \\
11(21 \%) \\
12(21 \%) \\
\text { not scored }(7 \%)\end{array}$ & $\begin{array}{c}7(2 \%) \\
8(13 \%) \\
9(15 \%) \\
10(24 \%) \\
11(33 \%) \\
12(13 \%)\end{array}$ & $\begin{array}{c}7(20 \%) \\
10(20 \%) \\
11(60 \%)\end{array}$ & $\begin{array}{c}11(60 \%) \\
12(20 \%) \\
\text { unscorable } \\
(20 \%)\end{array}$ & $\begin{array}{c}11(9 \%) \\
12(64 \%) \\
13(27 \%)\end{array}$ \\
\hline UppLab & $\begin{array}{c}7(94 \%) \\
8(6 \%)\end{array}$ & $7(100 \%)$ & $\begin{array}{c}7(16 \%) \\
8(79 \%) \\
9(5 \%)\end{array}$ & $\begin{array}{l}7(59 \%) \\
8(41 \%)\end{array}$ & $8(100 \%)$ & $\begin{array}{l}7(60 \%) \\
8(40 \%)\end{array}$ & $8(100 \%)$ \\
\hline EarL & $\begin{array}{c}3(63 \%) \\
4(31 \%) \\
5(6 \%)\end{array}$ & $\begin{array}{c}3(25 \%) \\
4(67 \%) \\
5(8 \%)\end{array}$ & $\begin{array}{l}3(26 \%) \\
4(63 \%) \\
5(11 \%)\end{array}$ & $\begin{array}{c}3(30 \%) \\
4(61 \%) \\
5(5 \%) \\
\text { not scored }(4 \%)\end{array}$ & $\begin{array}{l}3(73 \%) \\
4(27 \%)\end{array}$ & $\begin{array}{l}3(80 \%) \\
4(20 \%)\end{array}$ & $\begin{array}{l}3(86 \%) \\
4(14 \%)\end{array}$ \\
\hline EarLPos & $\begin{array}{l}1(40 \%) \\
2(60 \%)\end{array}$ & $\begin{array}{c}1(8 \%) \\
2(83 \%) \\
3(9 \%)\end{array}$ & $\begin{array}{l}1(5 \%) \\
2(84 \%) \\
3(6 \%) \\
4(5 \%)\end{array}$ & $\begin{array}{c}1(7 \%) \\
2(70 \%) \\
3(17 \%) \\
4(2 \%) \\
\text { not scored }(4 \%)\end{array}$ & $\begin{array}{l}1(27 \%) \\
2(64 \%) \\
3(9 \%)\end{array}$ & $\begin{array}{l}1(40 \%) \\
2(60 \%)\end{array}$ & $\begin{array}{l}1(86 \%) \\
2(14 \%)\end{array}$ \\
\hline
\end{tabular}

The unweighted parsimony analyses and the Bayesian analyses of the mitochondrial data recovered a wellresolved topology with strong support for relationships among major clades (Figure 2a). As expected, analyses of the nDNA data set produced a much more conservative topology (Figure 2b), but one that is highly concordant with the mtDNA tree. Figure 3 shows the results of the partitioned Bayesian analysis of the combined multi-gene data set. Results of the un-partitioned analyses were virtually identical and are not shown. The combined multigene analysis produced a well-resolved and well-supported topology that is very similar to both individual gene trees. We focus description of the phylogeny and the discussion on results from the combined multi-gene data set. 
The species C. labillardieri comprises seven well-supported clades (Figure 3) that occupy distinct geographic regions (Figure 1). There is relatively little support for the relationships between these $C$. labillardieri clades. The species $C$. catenifer, $C$. youngsoni, and $C$. gemmula are each very well supported with both $C$. catenifer and $C$. gemmula showing phylogeographic structure (Figure 3). The threatened species $C$. lancelini from Lancelin Island and the adjacent mainland is the sister taxon to three individuals that had been identified as C. labillardieri. Our molecular data demonstrate that these animals, from the Swan Coastal Plain, instead are more closely related to $C$. lancelini. We describe these animals, plus two additional specimens, as a new species below.

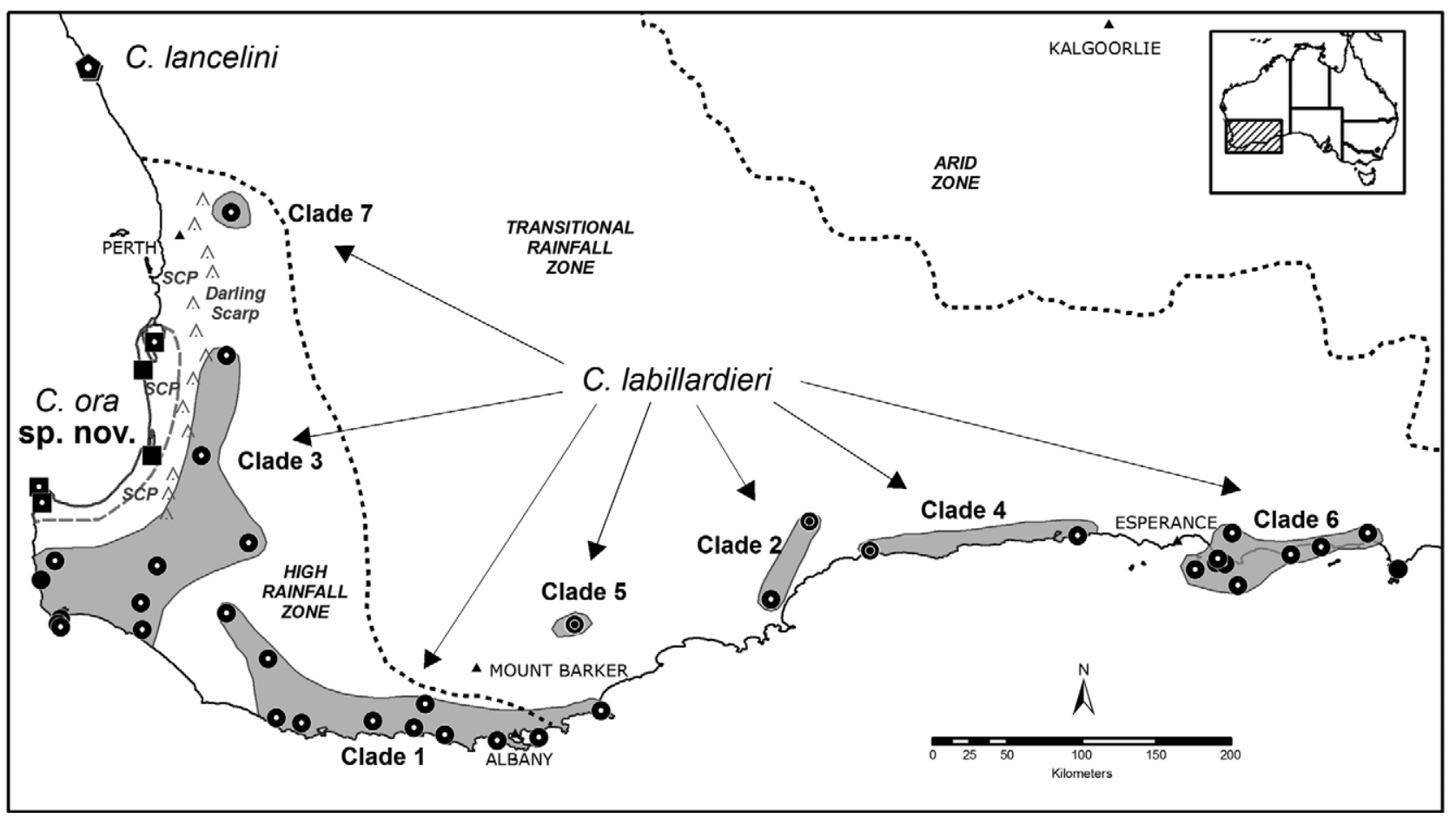

FIGURE 1. Distribution of the seven major $C$. labillardieri (circle) clades, the $C$. lancelini ( pentagonal) clade and the new taxon, $C$. ora sp. nov (square). Specimens used in both the genetic and morphological analyses are denoted with a white center, and specimens for which we only had morphological data are solid. The few specimens of $C$. labillardieri for which we only had tissue samples are denoted with an inner white ring. SCP refers to the Swan Coastal Plain west of the Darling Scarp. Black dotted lines represent boundaries between rainfall zones, as defined by Hopper (1979). Grey dotted line demarks the known range for C. ora sp. nov.

Morphological data. Table 6 summarizes the morphological measurements. As expected, there is considerable overlap between species in most of the continuous and categorical variables measured but the species vary consistently in body size, shape and colouration. We summarize the results of our PCA analysis on the continuously distributed characters in Figure 4 where we show mean PC scores and standard deviations. PC1 explains $51.8 \%$ of the variation, PC2 explains $10.5 \%$ of the variation and the mean PC scores varied significantly among $\operatorname{taxa}\left(\mathrm{PC} 1: \mathrm{F}_{6,132}=18.55, \mathrm{P}<0.0001 ; \mathrm{PC} 2: \mathrm{F}_{6,132}=11.70, \mathrm{P}<0.0001\right)$. As expected, the first principal component was very highly correlated with snout-vent length and thus body size $\left(\mathrm{SVL}, \mathrm{r}^{2}=0.91, \mathrm{P}<0.001\right)$ and the second principal component summarized shape and important scale differences among the species. Additional principal components explained negligible amounts of the variation. C. younsoni is the largest of the species, $C$. labilardieri and C. lancelini intermediate in size, and C. catenifer, C. delli, C. gemmula and C. ora sp. nov. (described below) the smallest (Figure 4, Table 6). C. younsoni is also the most divergent in terms of shape and meristics, $C$. labilardieri and $C$. lancelini are similar to each other in, and $C$. catenifer, $C$. delli and $C$. gemmula are extremely similar. C. ora sp. nov. (described below) is divergent from all the other species (Figure 4). Additional qualitative differences in coloration and color pattern are also important in distinguishing species.

Taxonomy. The genetic analysis provides a robust hypothesis of relationships among the species in the $C$. labillardieri species group and in addition demonstrates strong phylogeographic structure in C. labillardieri. Our detailed morphological analysis of all specimens included in our genetic work, and additional specimens, demonstrates that $C$. labillardieri is morphologically relatively homogeneous. Additional PCA of just C. labillardieri 
specimens did not show any clear patterns of morphological variation relative to the seven identified genetic clades (not shown), therefore we represent $C$. labilllardieri as one entity in Figure 4. Ctenotus labillardieri does display geographic variation in back patterns (Figure 5), but this variation also does not correspond to the seven identified genetic clades. Therefore, we treat $C$. labillardieri a single species. The one exception to this is a clade previously identified as C. labillardieri based on morphology, but our genetic data show that it instead is most closely related to $C$. lancelini. Our morphological data show that these animals are morphologically divergent from both $C$. labillardieri and C. lancelini (Figure 4; Table 6) and we describe it as a new species below. We examined photographs of the type specimen of Hinulia greyi Gray 1845, a name now in synonymy of C. labillardieri, and confirmed that this specimen is not the new taxon we describe here.
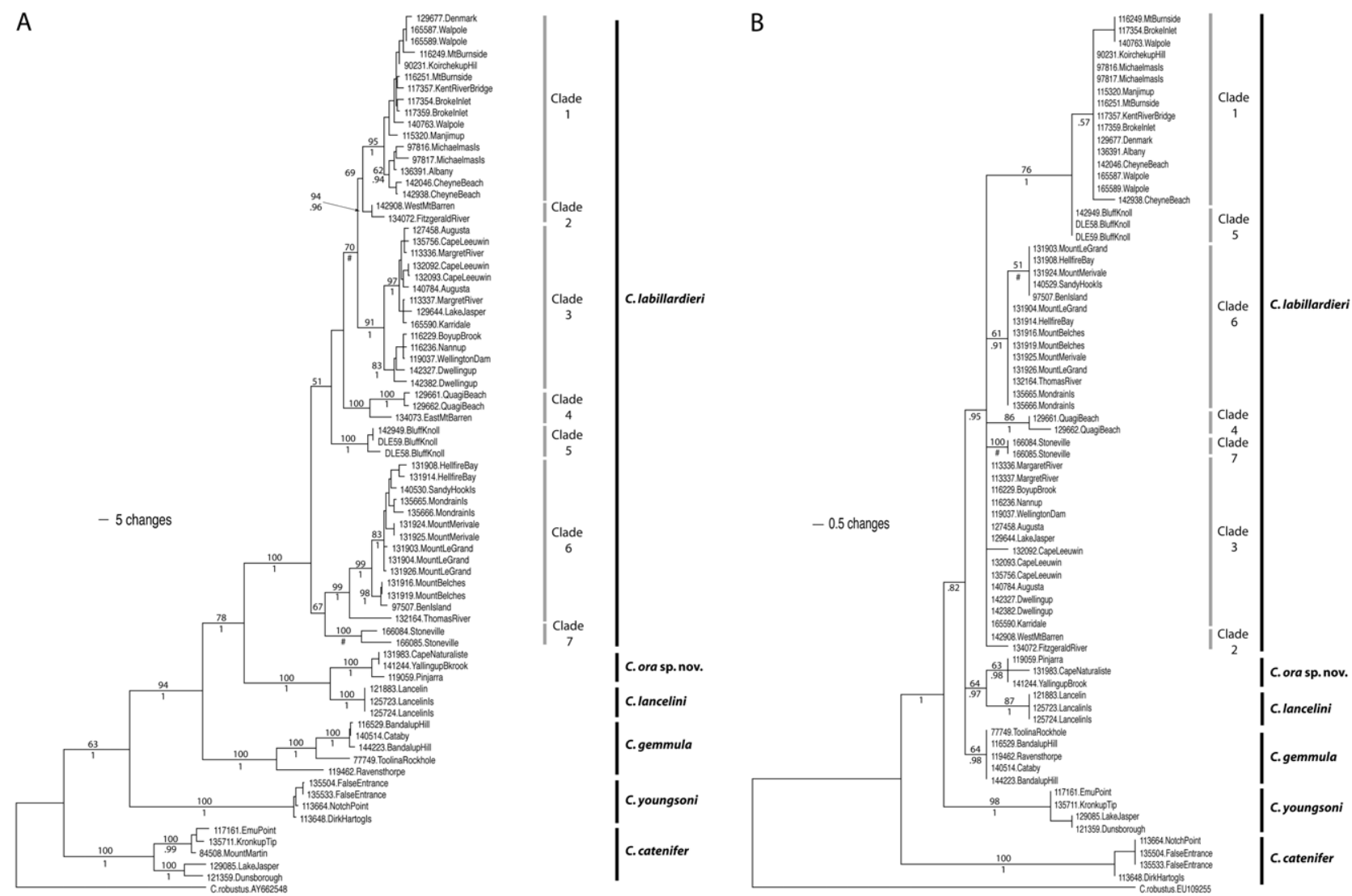

FIGURE 2. Individual gene trees for the two genes used in this study. A) Representative parsimony phylogram for the ND2 data set. B) Representative phylogram from the nDNA data set. Values on selected branches refer to parsimony bootstrap values above the branch and Bayesian posterior probabilities below.

\section{Ctenotus ora sp. nov.}

Coastal Plains Skink

(Figures 5j, 6, 7)

Holotype. WAM R131983. Type locality: Cape Naturaliste at 33³2`21"S, $115^{\circ} 01^{`} 13^{\prime \prime E}$. Collected by M. D. Shapiro on $4^{\text {th }}$ November 1997.

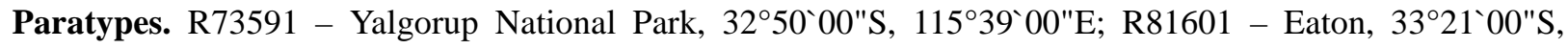
$115^{\circ} 42^{`} 00 " E$; R119059 - Lake Mealup (15km WSW Pinjarra), 3240`00"S, 11543`00"E; R141244 - Yallingup Brook, $33^{\circ} 38^{`} 39^{\prime \prime} \mathrm{S}, 115^{\circ} 02^{`} 15^{\prime \prime} \mathrm{E}$.

Diagnosis. Ctenotus ora is distinguished from sister taxon C. lancelini by its smaller size, generally darker colouration and lack of vertebral stripes (see Ford 1969). It is distinguishable from C. gemmula, C. delli and C. catenifer by a continuous white dorsolateral line, and from C. youngsoni by its smaller size and sharper dorsal patterning (Figure 5). C. ora can be distinguished from C. labillardieri by its smooth copper-brown dorsum and absence of white specks above the dorsolateral line. 


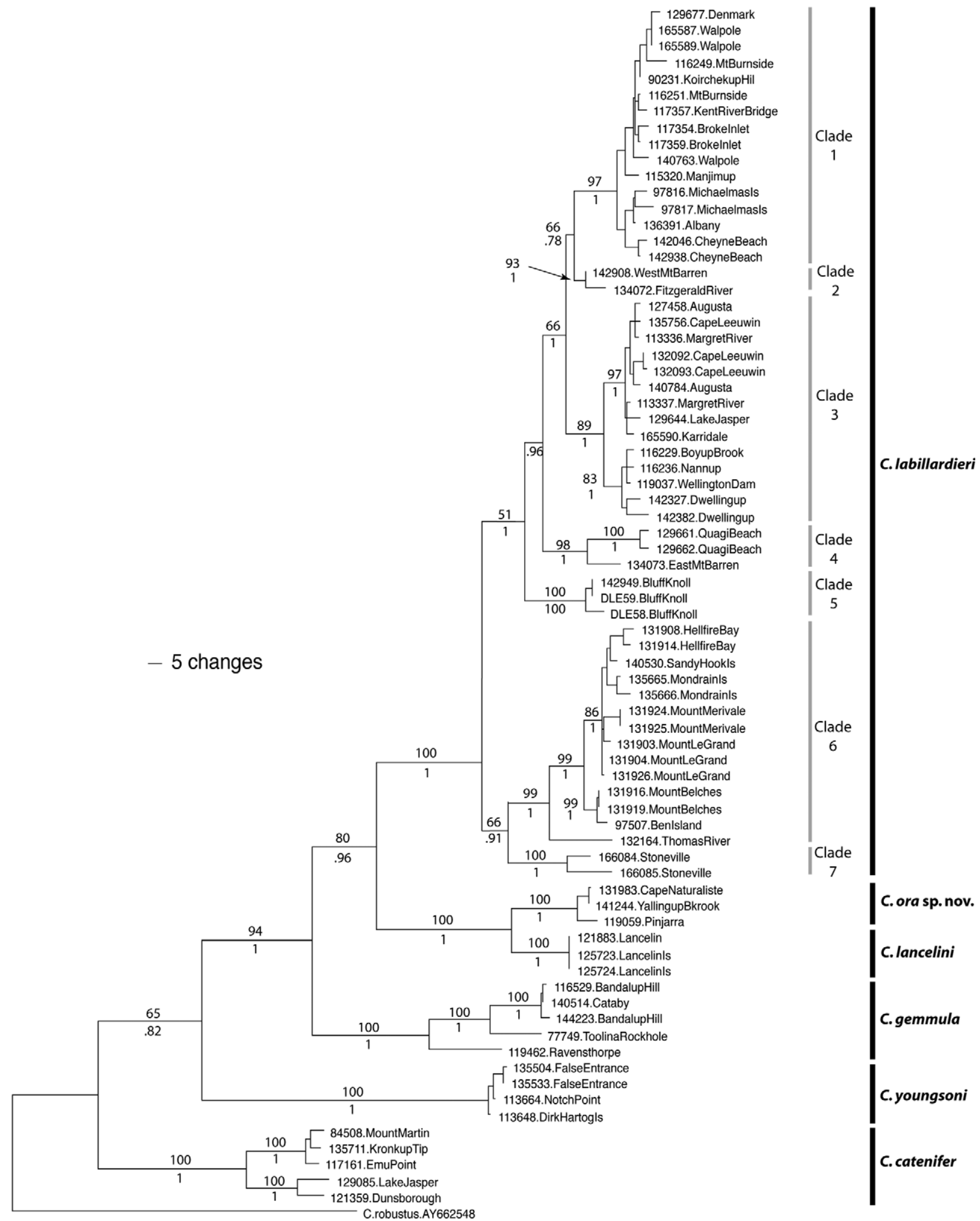

FIGURE 3. Phylogram based on analysis of the combined data set. Values on selected branches refer to parsimony bootstrap values above the branch and Bayesian posterior probabilities below.

Description. A small to medium-sized (maximum SVL 60mm) member of the Ctenotus labillardieri species group. Measurements for 19 morphological characters are summarized in Table 6. In addition to these: external ear opening prominent, small and ovate, about half the diameter of eye; snout triangular in profile with nose rounded; body slender, pentadactyl limbs; forelegs extend beyond the eye when adpressed; hindlimbs long, reaching beyond 
two-thirds of the axilla-groin length when adpressed; digits moderately long and slender; finger length: $4>3>2>5>1$; toe length: $4>3>5>2>1$; tail round in cross-section with very gradual taper to its pointed tip; head scales smooth; nasals separated; prefrontals separated; supraoculars four, with first two in contact with frontal; ear lobules three, occasionally four, with either the $1^{\text {st }}$ or $2^{\text {nd }}$ the largest.

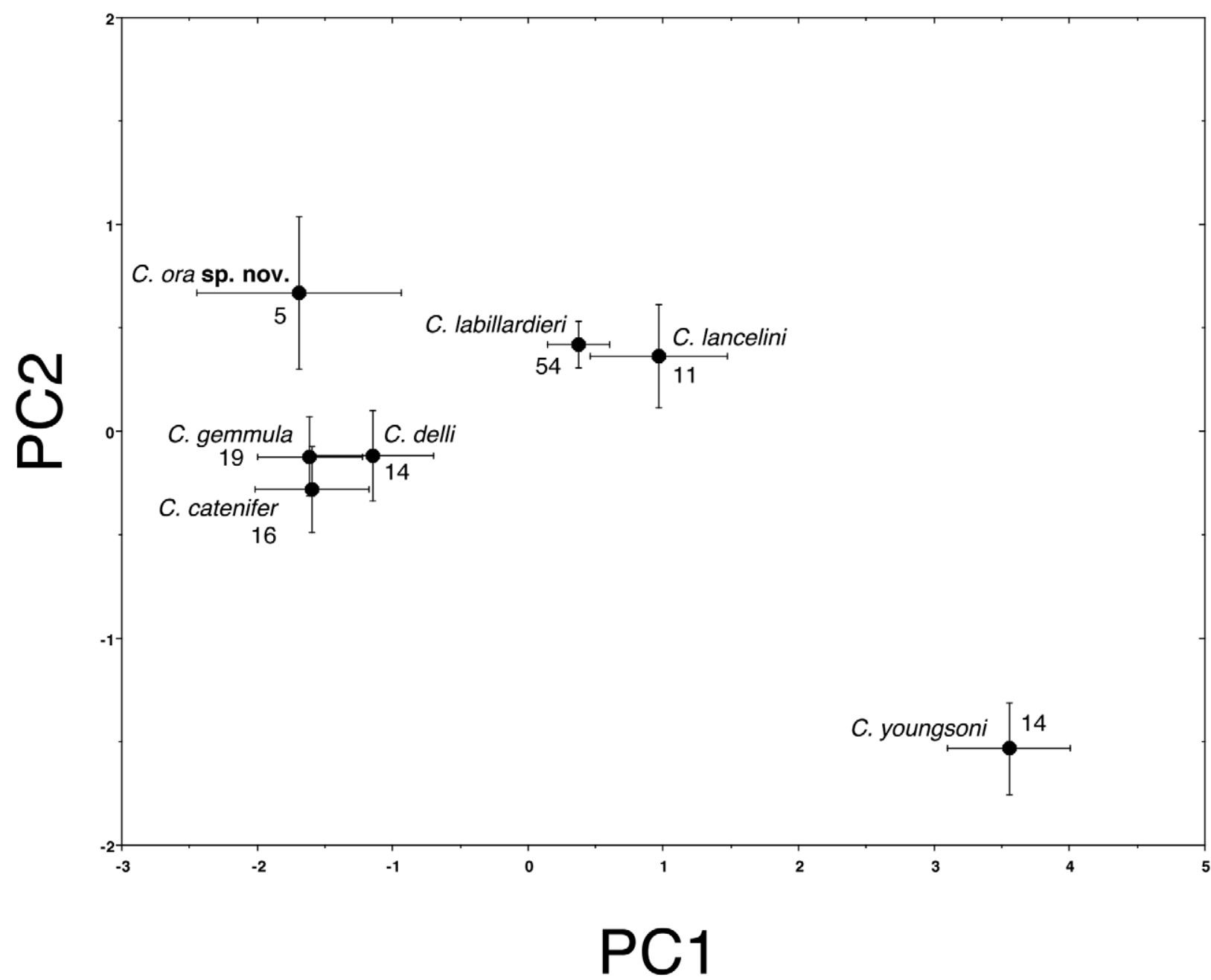

FIGURE 4. Summary of results for the principal component analyses (PCAs) of the morphological data. Mean principal component scores and standard deviations are shown with sample sizes noted.

Colouration. Dorsal surface bronze-brown, without any black pigmentation within the bronze-brown ground colour, creating a smooth appearance; white dorsolateral, midlateral and ventrolateral stripes, the latter much less sharp and defined than the former two; below each white stripe is a dark brown-black band, the most ventral of which is narrowest and least defined (Figure 5); some fine white flecks between the dorsolateral and upper lateral stripes; chin and throat uniform whitish-grey in preserved specimens; digital lamellae with slightly darker pigmentation; legs reddish-orange with black patches covering nearly half of each leg (Figure 7).

\section{Description of holotype.}

SVL - 58mm; HeadL - 14.4mm; HLL - 23mm; FLL - 15mm; ILL - 35mm; TailL - 88mm; LorH:W - .91; MidB - 22; Lam4Toe - 24; VentS - 48; NasSS - Yes; PrefSS - Yes; SOS - 4; SOSContact - 2; SupCil - 8; Palp 11; UppLab - 8; EarL - 3; EarLPos - 2.

Variation. Table 6 presents the morphological variation for the 19 characters measured. Juveniles show the same overall colour patterns, but with somewhat finer black blotching on legs. There appears to be little geographic variation.

Habitat. Specimen WAM R119059 was found under a banksia log in open eucalypt woodland over Banksia attenuata and Banksia grandis on white sand. Specimen WAM R73591 was found in Corymbia calophylla over heath in sandy soil. The species seems to have a preference for sandy substrates with low vegetation with open Eucalyptus woodland over Banksia (B. Maryan, pers. comm.) 
Distribution. This species appears to be restricted to the SWA coastal plain west of the Darling Range, south of Perth, Western Australia. In addition to the specimens examined and listed in Table 1, we examined photographs of specimens from Lake Clifton on the Swan Coastal Plain and these appear to also represent $C$. ora (WAM R17966-68). It is known to occur as far north as Pinjarra and south as far as Yallingup Brook, where it occupies coastal dunes. Across its range it occurs in very low densities, in contrast to neighbouring C. labillardieri populations, which are found in great abundance throughout the Darling Range (B. Maryan, pers. comm.).
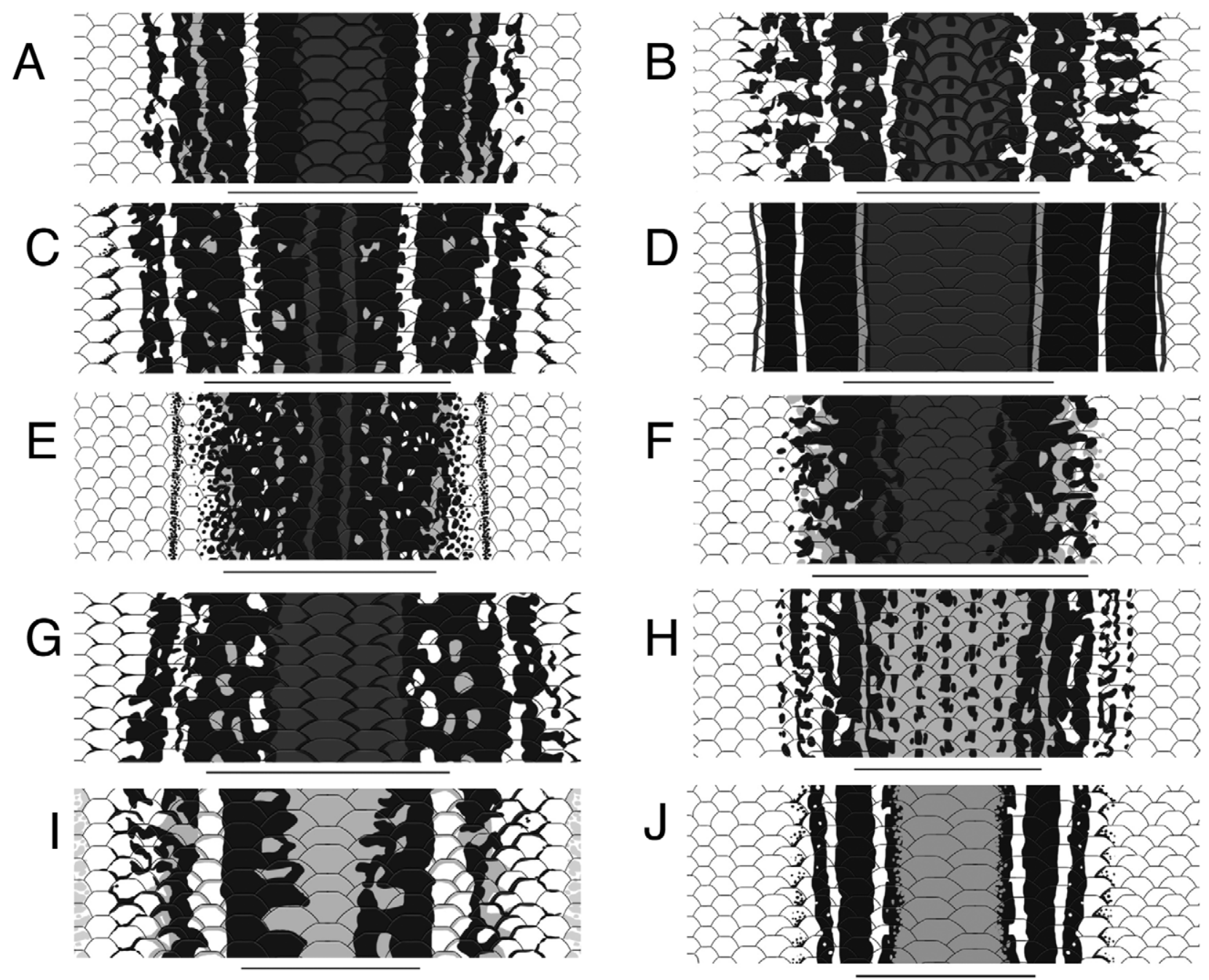

FIGURE 5. Back patterns in the Ctenotus labillardieri species group. A-D illustrate geographic variation in C. labillardieri and E-J show representative back patterns in the other species in the group. The variation shown within C. labillardieri is not confined to the clades illustrated. A) C. labillardieri (R135665; lineage 6), B) C. labillardieri (R117354, lineage 1), C) C. labillardieri (R142908, lineage 2), D) C. labillardieri (166085, lineage 7), E) C. catenifer (R163143), F) C. delli (R13444), G) C. gemmula (R150245), H) C. lancelini (R18874), I) C. youngsoni (R66208), J) C. ora sp. nov. (R131983). Scale bars are all 1cm.

Etymology. ora is Latin for "coast", "seaside" or "shore" and is in reference to the coastal distribution of the species.

Similar species. Despite large morphological variation within C. labillardieri in color patterns, C. ora can be readily distinguished by its smooth copper-brown dorsum and absence of white specks above the dorsolateral line. All C. labillardieri (with the exception of Clade 2) shared varying degrees of melanism on the dorsal surface scales, creating either an unconnected pattern of dark flecks, or a connected set of one or two vertebral stripes (Figure 5). Furthermore, $C$. ora lacks the heavily speckled flanks present in all $C$. labillardieri clades, with the exception of $C$. labillardieri Clade 2. Instead, both $C$. ora and C. labillardieri Clade 2 possess two dark brown and one white ventrolateral stripes with Clade 2 being distinguished from $C$. ora by the most ventral brown zone much more solid and defined. In addition, $C$. labillardieri Clade 2 lacks any white specks between the dorso-lateral and mid-lateral stripes, giving these individuals a uniquely "immaculate" appearance overall, in contrast to the subtle 
white speckling in $C$. ora. Finally, the ventral surface of $C$. ora is whitish and clean where $C$. labillardieri Clade 2 individuals have dark flecks under the chin and throat.

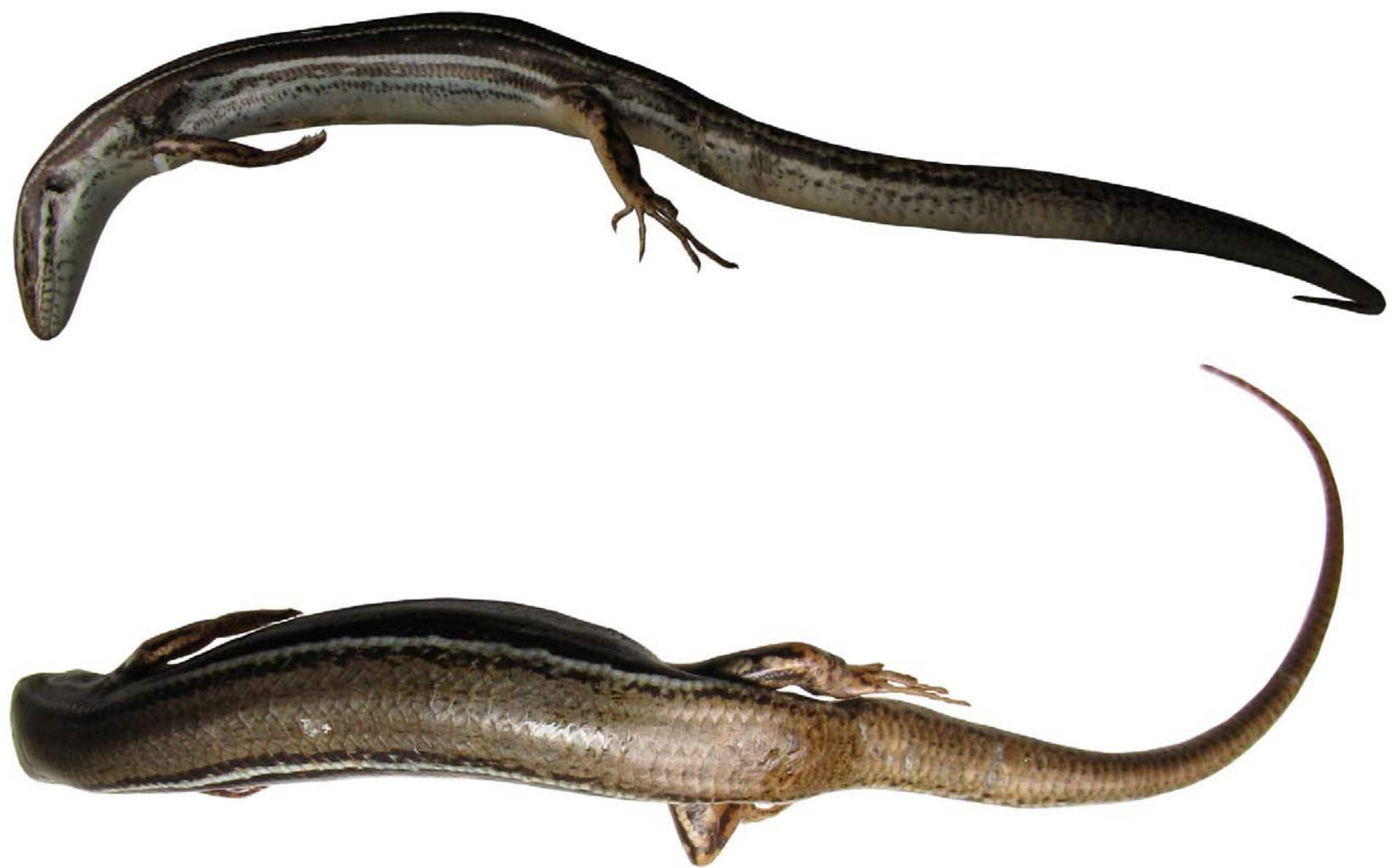

FIGURE 6. Photographs of the holotype of Ctenotus ora sp. nov. (WAM R131983).

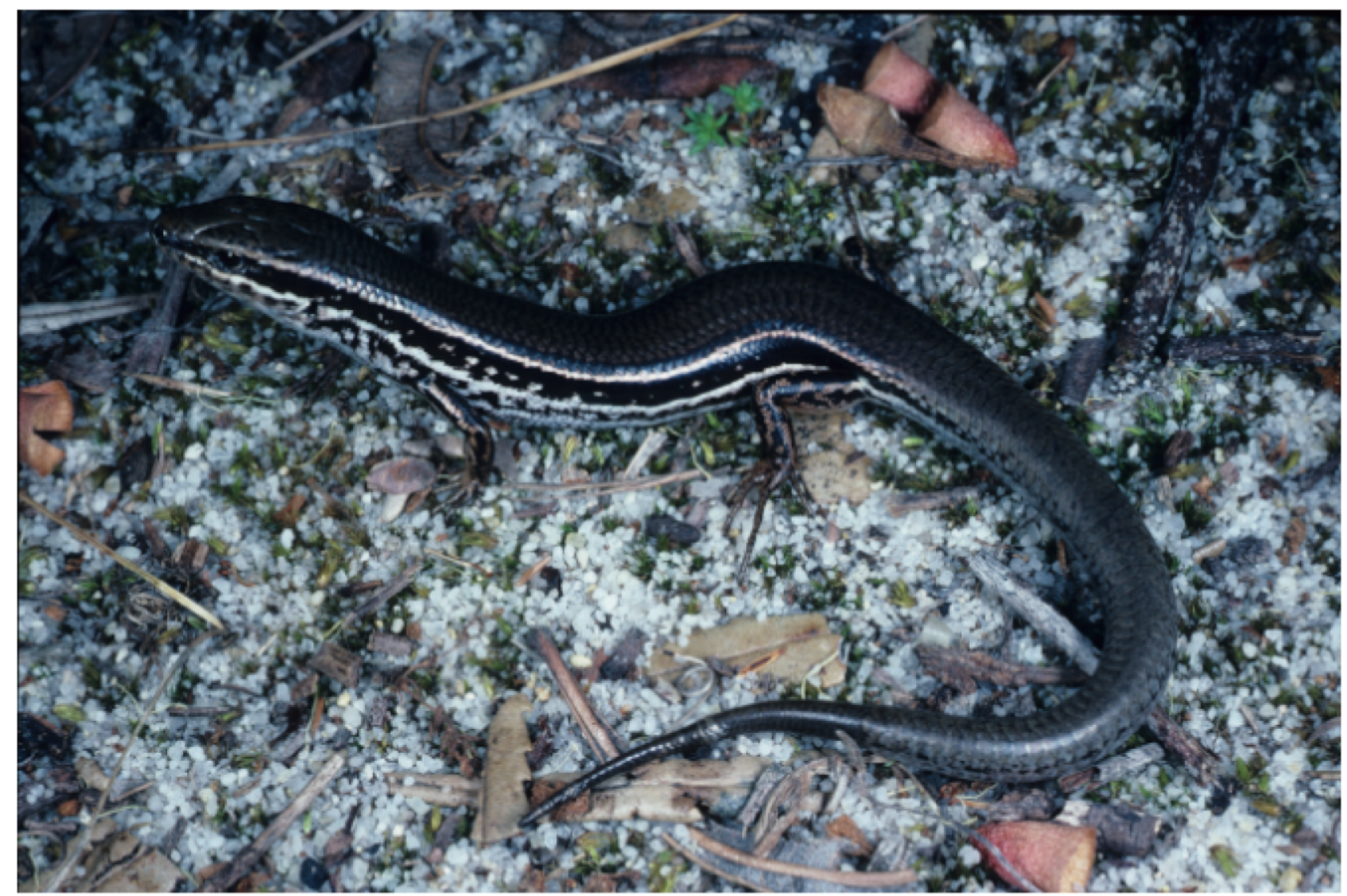

FIGURE 7. Photograph in life of Ctenotus ora sp. nov. from Pinjarra, Western Australia (WAM R119059). 


\section{Discussion}

\section{Molecular phylogeny of the $C$. labillardieri species group}

A morphological revision of Ctenotus by Storr (1974), which included C. labillardieri, resulted in the recognition of several new species, including C. catenifer, C. delli, and C. gemmula, which together with C. youngsoni and the threatened C. lancelini form the larger 'labillardieri group' (Storr 1974; Storr et al. 1999). Our molecular data provides the first detailed phylogeny for these taxa and strong support for the species status of all members for which we could obtain tissues (no tissues available for $C$. delli). Our molecular and morphological data further provide important information on phylogeographic structuring and morphological variation in C. labillardieri, the first such published data set for a reptile in SWA, and evidence for a new species of Ctenotus. Each of the species in the C. labillardieri species group is 10-18\% divergent in mtDNA from the other species, with the exception of $C$. lancelini and $C$. ora sp. nov., which are approximately $4 \%$ divergent. We first outline the phylogeographic structure and morphological variation in C. labillardieri, followed by some brief comments on C. catenifer and C. gemmula. Lastly, we consider the newly described species $C$. ora $\mathbf{s p . ~ n o v . ~}$

\section{Phylogeography of $C$. labillardieri clades}

Our molecular phylogeny shows that $C$. labillardieri displays considerable intraspecific genetic diversity with strong support for seven genetic clades (Figure 1,3). The genetic distances between many of these clades (Table 4 and 5) are equivalent to species-level differences in other reptiles using mitochondrial genes that evolve at a similar rate (e.g. ND4, Scott \& Keogh 2000), but importantly, our morphological data show high levels of geographic variation that do not correspond well to the genetic patterns. Therefore, we maintain that $C$. labillardieri should be considered a single but morphologically variable species.

Clades 1, 3 and 7 are restricted to the High Rainfall Zone (HRZ) of southwestern Australia. The biogeographic break between Clades 1 and 3 coincides with boundaries recognised in multiple taxonomic groups, including plants (Lambertia orbifolia [ Byrne et al. 1999]), frogs (Geocrinia rosea species complex [Wardell-Johnson \& Roberts 1993]; Geocrinia leai [Edwards 2007b]; Metacrinia nichollsi [Edwards et al. 2008]) and freshwater invertebrates (Engaewa subcoerulea and E. similis [Horwitz \& Adams 2000]; Cherax preissii [Gouws et al. 2006]). Contraction to moist refugia during arid pulses of the Pliocene/Pleistocene (Dodson \& Macphail 2004) is thought to have promoted much of this divergence. This seems like a plausible explanation for the patterns seen in our study given the obvious ecological preference of $C$. labillardieri, where dependence on wet restricted sites such as granite outcrops is likely to have resulted in limited gene flow between populations during arid periods. Indeed, a previous study of C. labillardieri suggested that the isolation of populations to granite outcrops accounted for observed morphological differences throughout SWA (Ford 1969). However, habitat differentiation may also be important for diversification in this taxon. For example, the distribution of Clade 1 appears to largely coincide with the Warren biogeographic subregion, defined under the Interim Biogeographical Regionalisation of Australia (IBRA), further supporting its recognition as a distinct bioregion (Thackway \& Cresswell 1995). In addition, the eastern extent of the distribution of Clade 1 coincides with the Transitional Rainfall Zone (TRZ) / HRZ climatic boundary, recognized as a significant climatic barrier for plants (Hopper 1979; Hopper \& Gioia 2004) and frogs (Edwards et al. 2007, 2008). However, the close relationship between Clade 2 in the TRZ and Clades 1 and 3 in the HRZ suggests a more recent genetic connection with subsequent geographic isolation across the TRZ / HRZ boundary. Both Clades 4 and 5 appear to be geographic isolates within the TRZ. This is consistent with climatic data suggesting long-term geographic isolation as the primary driver for the huge diversity of endemic species and populations found in the Stirling Ranges (Clade 5) (Erika et al. 1993; Main 1996; Hopper \& Gioia 2004; Edwards et al. 2008; Rix \& Harvey 2012). Similarly, Clade 4 may have experienced a prolonged period of geographic isolation based on strong support by the nuclear data. This is consistent with patterns in plants (Byrne \& Hines 2004) and frogs (Edwards et al. 2007a) which implicate a xeric barrier east of the Bremer Bay region as the primary cause for this isolation. The distributions of clades 2 and 4 also fall within different IBRA subregions of the Esperance Plains; the Fitzgerald (Clade 2) and Recherche (Clade 4). Both regions comprise subdued coastal sandplains on the coast, but differ in environmental aspects such as rainfall and geological substrate (Environment Australia 2004).

\section{Biogeographic comments on $C$. catenifer and $C$. gemmula}

While limited sampling precludes any detailed phylogeographic comments, we found substantial genetic structure in C. catenifer and C. gemmula (Table 4). Interestingly, the two clades recovered in C. catenifer have geo- 
graphic distributions much like $C$. labillardieri clades 1 and 3, adding further support that this region has historically been an important biogeographic barrier to moist-adapted taxa. Our sampling of C. gemmula recovered two divergent individuals, each represented by a single individual. A third clade contains closely related individuals from Cataby near Perth, and Bandalup, west of Esperance. This pattern is remarkably similar to C. labillardieri clades 6 and 7 (albeit with low support in C. labillardieri) suggesting recent connectivity despite large geographic distances across the TRZ.

\section{Ctenotus ora, a new species of immediate conservation concern}

Ctenotus ora sp. nov. comprises specimens that previously were allocated to the widely distributed and morphologically variable $C$. labillardieri. Our genetic data demonstrate that it instead is the sister taxon to the threatened C. lancelini. Ctenotus lancelini is distributed only on Lancelin Island and the immediate adjacent mainland. A recovery plan for this species outlined unpublished allozyme data (Mark Adams, South Australian Museum) and morphological evaluation (Ken Aplin, Western Australian Museum) which suggested 'the existence of a taxon closely related or the same as $C$. lancelini' and made a call for detailed genetic and morphological data to sort out this problem (Pearson \& Jones 2000). Our molecular and morphological data resolve this issue and demonstrate that $C$. lancelini and $C$. ora sp. nov. are closely related but genetically and morphological quite distinct species, each of which are habitat specialists. Ctenotus lancelini is threatened and a recovery plan is in place for its longterm survival (Pearson \& Jones 2000). Ctenotus ora sp. nov. is restricted in distribution to the sandy Swan Coastal Plain subregion south of Perth, a region now under heavy development from population growth from Perth, and an area of biogeographic significance (Horwitz \& Adams 2000; Wheeler \& Byrne 2006). Ctenotus ora sp. nov. is known to be very sparsely distributed in the region and may already be under threat. The conservation status of this new species should be assessed immediately.

\section{Acknowledgements}

We thank Brad Maryan, Danielle Edwards, Conrad Hoskin, Suzi Morrison, and David Moore for assistance on field collecting trips and in genetic analysis. All tissue samples and vouchers examined came from the Western Australian Museum and we are indebted to Paul Doughty, Brad Maryan, and Claire Stevenson for assistance. We especially thank Glenn Shea and Paul Doughty for photographs of type series and other animals and also for critically reading the manuscript and providing many helpful comments. We thank Erin Walsh for the beautiful drawings of the lizard back patterns and Mitzy Pepper for fine tuning. Funding was contributed by the Australian Greenhouse Office (GK) and the Australian Research Council (JSK).

\section{References}

Beard, J.S., Chapman, A.R. \& Gioia, P. (2000) Species richness and endemism in the Western Australian flora. Journal of Biogeography, 27, 1257-1268.

Berry, O. (2001) Genetic evidence for wide dispersal by the sand frog, Heleioporus psammophilus (Anura: Myobatrachidae), in Western Australia. Journal of Herpetology, 35, 136-141.

Bush, B., Maryan, B., Browne-Cooper, R. \& Robinson, D. (2007) Reptiles and Frogs in the Bush. Perth, Australia: University of Western Australia Press.

Byrne, M. (2007) Phylogeography provides an evolutionary context for the conservation of a diverse and ancient flora. Australian Journal of Botany, 55, 316-325.

Byrne, M. \& Hines, B. (2004) Phylogeographical analysis of cpDNA variation in Eucalyptus loxophleba (Myrtaceae). Australian Journal of Botany, 52, 459-470.

Byrne, M. \& Hopper, S.D. (2008) Granite outcrops as ancient islands in old landscapes: evidence from the phylogeography and population genetics of Eucalyptus caesia (Myrtaceae) in Western Australia. Biological Journal of the Linnean Society, 93, $177-188$.

Byrne, M., Macdonald, B. \& Brand, J. (2003a) Phylogeography and divergence in the chloroplast genome of Western Australian Sandalwood (Santalum spicatum). Heredity, 91, 389-395.

Byrne, M., MacDonald, B., Broadhurst, L. \& Brand, J. (2003b) Regional genetic differentiation in Western Australian sandalwood (Santalum spicatum) as revealed by nuclear RFLP analysis. Theoretical and Applied Genetics, 107, $1208-1214$. 
Byrne, M., Macdonald, B. \& Coates, D. (1999) Divergence in the chloroplast genome and nuclear rDNA of the rare Western Australian plant Lambertia orbifolia Gardner (Proteaceae). Molecular Ecology, 8, 1789-1796.

Cincotta, R.P., Wisnewski, J. \& Engelman, R. (2000) Human population in the biodiversity hotspots. Nature, 404, 990-992.

Cooper, S.J.B., Harvey, M.S., Saint, K.M., \& Main B.Y. (2011) Deep phylogeographic structuring of populations of the trapdoor spider Moggridgea tingle (Migidae) from southwestern Australia: evidence for long-term refugia within refugia. Molecular Ecology, 20, 3219-3236.

Dodson, J.R. \& Macphail, M.K. (2004) Palynological evidence for aridity events and vegetation change during the Middle Pliocene, a warm period in Southwestern Australia. Global and Planetary Change, 41, 285-307.

Driscoll, D.A. (1998a) Genetic structure of the frogs Geocrinia lutea and Geocrinia rosea reflects extreme population divergence and range changes, not dispersal barriers. Evolution, 52, 1147-1157.

Driscoll, D.A. (1998b) Genetic structure, metapopulation processes and evolution influence the conservation strategies for two endangered frog species. Biological Conservation, 83, 43-54.

Edwards, D.L. (2007a) Biogeography and speciation of a direct developing frog from the coastal arid zone of Western Australia. Molecular Phylogenetics and Evolution, 45, 494-505.

Edwards, D.L. (2007b) Biogeography and speciation of southwestern Australian frogs, PhD Thesis. The University of Western Australia, Perth, Western Australia.

Edwards, D.L., Roberts, J.D. \& Keogh, J.S. (2007) Impact of Plio-Pleistocene arid cycling on the population history of a southwestern Australian frog. Molecular Ecology, 16, 2782-2796.

Edwards, D.L., Roberts, J.D. \& Keogh, J.S. (2008) Climatic fluctuations shape the phylogeography of a mesic direct-developing frog from the south-western Australian biodiversity hotspot. Journal of Biogeography, 35, 1803-1815.

Environment Australia (2004) Revision of the Interim Biogeographic Regionalisation of Australia (IBRA) and the Development of Version 6.1. - Summary Report. Department of Sustainability, Environment, Water, Populations, and Communities. Canberra.

Erika, P., Sandro, P. \& Fernando, L. (1993) Plant communities of the Stirling Range, Western Australia. Journal of Vegetation Science, 4, 477-488.

Ford, J. (1969) Distribution and variation of the skink Ctenotus labillardieri (Grey) of southwestern Australia. Western Australian Naturalist, 7, 68-75.

Gouws, G., Stewart, B.A. \& Daniels, S.R. (2006) Phylogeographic structure of a freshwater crayfish (Decapoda : Parastacidae : Cherax preissii) in south-western Australia. Marine and Freshwater Research, 57, 837-848.

Hopper, S.D. (1979) Biogeographical aspects of speciation in the southwest Australian flora. Annual Review of Ecology and Systematics, 10, 399-422.

Hopper, S.D. \& Gioia, P. (2004) The Southwest Australian Floristic Region: Evolution and conservation of a global hot spot of biodiversity. Annual Review of Ecology Evolution and Systematics, 35, 623-650.

Horwitz, P. \& Adams, M. (2000) The systematics, biogeography and conservation status of species in the freshwater crayfish genus Engaewa Riek (Decapoda : Parastacidae) from south-western Australia. Invertebrate Taxonomy, 14, 655-680.

Huelsenbeck, J. P. \& Ronquist, F. (2001) MRBAYES: Bayesian inference of phylogenetic trees. Bioinformatics, 17, 754-755.

Main, B.Y. (1996) Terrestrial invertebrates in south-west Australian forests: the role of relict species and habitats in reserve design. Journal of the Royal Society of Western Australia, 79, 277-280.

Main, B.Y. (1999) Notes on the biogeography and natural history of the orbweaving spider Carepalxis (Araneae, Araneidae), including a gumnut mimic from southwestern Australia. Journal of Arachnology, 27, 183-188.

Morgan, M., Roberts, J.D. \& Keogh, J.S. (2007) Molecular phylogenetic dating supports an ancient endemic speciation model in Australia's biodiversity hotspot. Molecular Phylogenetics and Evolution, 44, 371-385.

Myers, N., Mittermeier, R.A., Mittermeier, C.G., da Fonseca, G.A.B. \& Kent, J. (2000) Biodiversity hotspots for conservation priorities. Nature, 403, 853-858.

Pearson, D. \& Jones, B. (2000) Lancelin Island Skink Recovery Plan. In: Department of Conservation and Land Management (Ed). Western Australian Wildlife Management Program.

Pepper, M., Doughty, P. \& Keogh, J.S. (2006) Molecular phylogeny and phylogeography of the Australian Diplodactylus stenodactylus (Gekkota; Reptilia) species-group based on mitochondrial and nuclear genes reveals an ancient split between Pilbara and non-Pilbara D. stenodactylus. Molecular Phylogenetics and Evolution, 41, 539-555.

Pepper, M., Doughty P., Arculus R., Keogh J.S. (2008) Landforms predict phylogenetic structure on one of the world's most ancient surfaces. BMC Evolutionary Biology, 8,152.

Rabosky, D. L., Donnellan, S.C., Talaba, A.L. \& Lovette, I.J. (2007) Exceptional among-lineage variation in diversification rates during the radiation of Australia's most diverse vertebrate clade. Proceedings of the Royal Society B-Biological Sciences, 274, 2915-2923.

Rix, M.G. \& Harvey, M.S. (2012) Phylogeny and historical biogeography of ancient assassin spiders (Araneae:Archaeidae) in the Australian mesic zone: Evidence for Miocene speciation within Tertiary refugia. Molecular Phylogenetics and Evolution, 62, 375-396.

Scott, I.A. \& Keogh, J.S. (2000) Conservation genetics of the endangered grassland earless dragon Tympanocryptis pinguicolla (Reptilia: Agamidae) in Southeastern Australia. Conservation Genetics, 1, 357-363.

Skinner, A. (2007) Phylogenetic relationships and rate of early diversification of Australian Sphenomorphus group scincids (Scincoidea, Squamata). Biological Journal of the Linnean Society, 92, 347-366. 
Storr, G.M. (1974) The genus Ctenotus (Lacertilia, Scincidae) in the South-West and Eucla Divisions of Western Australia. Journal and Proceedings for the Royal Society of Western Australia, 56, 89-93.

Storr, G.M., Smith, L.A. \& Johnstone, R.E. (1999) Lizards of Western Australia - I. Skinks (Second Edition ed.): University of Western Australia Press with Western Australian Museum.

Swofford, D. (2002) PAUP*. Phylogenetic Analysis Using Parsimony (*and Other Methods). Sunderland, MA.: Sinauer Associates.

Thackway, R. \& Cresswell, I. (1995) An Interim Biogeographic Regionalisation for Australia: a framework for setting priorities in the National Reserves System Cooperative Program. Canberra: Reserve System Unit, Australian Nature Conservation Agency.

Wardell-Johnson, G. \& Roberts, J.D. (1993) Biogeographic Barriers in a Subdued Landscape - the Distribution of the Geocrinia rosea (Anura, Myobatrachidae) Complex in South-Western Australia. Journal of Biogeography, 20, 95-108.

Wheeler, M.A. \& Byrne, M. (2006) Congruence between phylogeographic patterns in cpDNA variation in Eucalyptus marginata (Myrtaceae) and geomorphology of the Darling Plateau, south-west of Western Australia. Australian Journal of Botany, 54, 17-26.

Wilson, S. \& Swan, G. (2008) A complete guide to reptiles of Australia (2nd Edition ed.). Sydney: New Holland Publishers (Australia) Pty Ltd.

Yates, C.J., Ladd, P.G., Coates, D.J. \& McArthur, S. (2007) Hierarchies of cause: understanding rarity in an endemic shrub Verticordia staminosa (Myrtaceae) with a highly restricted distribution. Australian Journal of Botany, 55, 194-205. 